\title{
Article \\ Current Status of Cardiac Rehabilitation in the Regional Cardiocerebrovascular Centers in Korea
}

\author{
Chul Kim ${ }^{1, *}$, Jidong Sung ${ }^{2}$, Jae-Young Han ${ }^{3}{ }^{-}$, Sungju Jee ${ }^{4}$, Jang Woo Lee ${ }^{5}{ }^{(}$, Jong Hwa Lee ${ }^{6}$, \\ Won-Seok Kim ${ }^{7} \mathbb{D}^{\text {, Heui Je Bang }}{ }^{8}{ }^{(\mathbb{D}}$, Sora Baek ${ }^{9}$, Kyung-Lim Joa ${ }^{10}$, Ae Ryoung Kim ${ }^{11}{ }^{\mathbb{D}}$, So Young Lee ${ }^{12}$, \\ Jihee Kim ${ }^{13}$, Chung Reen Kim ${ }^{14}{ }^{(1)}$ and Oh Pum Kwon ${ }^{7(1)}$
}

check for

updates

Citation: Kim, C.; Sung, J.; Han, J.-Y.; Jee, S.; Lee, J.W.; Lee, J.H.; Kim, W.-S.; Bang, H.J.; Baek, S.; Joa, K.-L.; et al. Current Status of Cardiac

Rehabilitation in the Regional Cardiocerebrovascular Centers in Korea. J. Clin. Med. 2021, 10, 5079. https://doi.org/10.3390/jcm10215079

Academic Editors: Francesco Giallauria and Toshiaki Nakajima

Received: 24 September 2021

Accepted: 26 October 2021

Published: 29 October 2021

Publisher's Note: MDPI stays neutral with regard to jurisdictional claims in published maps and institutional affiliations.

Copyright: (c) 2021 by the authors. Licensee MDPI, Basel, Switzerland. This article is an open access article distributed under the terms and conditions of the Creative Commons Attribution (CC BY) license (https:// creativecommons.org/licenses/by/ $4.0 /)$.
1 Department of Rehabilitation Medicine, Inje University Sanggye Paik Hospital, Seoul 01757, Korea

2 Division of Cardiology, Department of Medicine, Sungkyunkwan University School of Medicine, Seoul 06351, Korea; jidong.sung@gmail.com

3 Department of Physical Medicine and Rehabilitation, Chonnam National University Medical School and Hospital, Gwangju 61469, Korea; rmhanjy@daum.net

4 Department of Rehabilitation Medicine, Chungnam National University College of Medicine, Daejeon 35015, Korea; drjeesungju@cnuh.co

5 Department of Physical Medicine and Rehabilitation, National Health Insurance Service Ilsan Hospital, Goyang 10444, Korea; medipia@gmail.com

6 Department of Physical Medicine and Rehabilitation, Dong A University College of Medicine, Busan 49201, Korea; jhlee08@dau.ac.kr

7 Department of Rehabilitation Medicine, Seoul National University Bundang Hospital, Seongnam 13620, Korea; Wondol77@gmail.com (W.-S.K.); Pum78@naver.com (O.P.K.)

8 Department of Rehabilitation Medicine, Chungbuk National University Hospital, Cheongju 28644, Korea; bang@chungbuk.ac.kr

9 Department of Rehabilitation Medicine, Kangwon National University School of Medicine, Chuncheon 24289, Korea; sora.baek@kangwon.ac.kr

10 Department of Rehabilitation Medicine, Inha University Hospital, Incheon 22332, Korea; drjoakl@gmail.com

11 Department of Rehabilitation Medicine, School of Medicine Kyungpook National University, Daegu 41944, Korea; Ryoung20@hanmail.net

12 Department of Rehabilitation Medicine, Jeju National University School of Medicine, Jeju 63241, Korea; bluelsy900@hanmail.net

13 Department of Rehabilitation Medicine, Wonkwang University School of Medicine, Iksan 54538, Korea; gold82mouse@hanmail.net

14 Department of Rehabilitation Medicine, Ulsan University College of Medicine, Ulsan 44033, Korea; crkim@uuh.ulsan.kr

* Correspondence: josephck@naver.com; Tel.: +82-2-950-1145

\begin{abstract}
Regional Cardiocerebrovascular Centers (RCCs)—a Korean government initiative—seek to reduce medical gaps across regions, and their cardiac rehabilitation (CR) programs are expected to model post-acute care for the Korean CR program. Accordingly, this study aimed to evaluate the current status of CR programs in the RCCs. We distributed surveys on the CR condition, activity, and barriers to 12 RCCs in different provinces. The results revealed significant gaps in the annual number of acute myocardial infarction admissions, and CR candidates, capacity, and density across the 12 RCCs. The CR capacity (50-500) and density (0.42-7.36) indicated particularly large gaps. Twelve RCCs had the necessary facilities, equipment and personnel for CR assessments and management, with high CR referral (97\%) and patient education (78\%) rates. However, the inpatient CR exercise training (56\%) participation rate was inadequate, with much lower enrollment $(47 \%)$ and adherence $(17 \%)$ rates to the outpatient CR program and large differences across centers. Therefore, this study's results will provide the evidence required to establish special national health strategies to overcome the CR barriers of patient, doctor/hospital, and policy factors for activating Korean CR programs.
\end{abstract}

Keywords: barriers; cardiac rehabilitation; hospital-based; participation rate 


\section{Introduction}

Cardiovascular disease (CVD) is the leading cause of mortality, morbidity, and healthcare costs in South Korea [1]. Cardiovascular (CV) mortality has risen by $42.8 \%$ over the last decade. It has become the second leading cause of death in South Korea [2]. According to the 2019 statistical office data, there were 62.42 deaths due to CVD per 100,000 individuals [3]. The modifiable CV risk factors' prevalence, such as smoking, dyslipidemia, obesity, and a sedentary lifestyle have continued to increase. The population is rapidly aging, with those aged 65 years and older projected to reach $37 \%$ by 2050 [1,4]. Death at the time of disease incidence, recurrence, and complications during follow-ups contribute to high mortality. There are also problems with decreased exercise capacity and quality of life. In addition, although most cases of CVD manifest clinically as an acute disease, they are actually chronic degenerative diseases that progress over a period of time. They thus need to be treated and managed as a chronic disease after discharge from the hospital [5].

According to the Organization for Economic Co-operation and Development (OECD) Reviews of Health Care Quality of Korea 2012, the "paradox" of South Korea was that casefatality rates were higher for acute myocardial infarction (AMI) than for OECD countries [4]. This is despite having excellent acute hospital care. This was largely due to inadequate CVD risk factor prevention and suboptimal emergency response systems. Additional contributors are inadequate post-acute care and marked disparities between rural and urban communities. This "paradox" is likely to be reinforced by the insufficiency of cardiac rehabilitation (CR) services, leading to a higher number of readmitted patients [4].

The Ministry of Health and Welfare (MHW) of South Korea has allocated government budgets from 2008 to install Regional Cardiocerebrovascular Centers (RCCs) in each province's main cities, except for Seoul. Fourteen RCCs have been installed until the present (Figure 1). The RCC project's goal was to minimize the incidence of complications and mortality. It also intends to reduce the medical gaps among the South Korean regions. This is conducted through timely medical service provision across the country. It also aims to facilitate patients' earlier return to society after their complete recovery [6]. At the RCCs, standardized and high-quality care for AMI and stroke has been established. They are required to be equipped with CR facilities, equipment, and personnel. They also have regular performance measures conducted by the Korea Disease Control and Prevention Agency (KDCA) on behalf of the MHW (Table 1). As a result, the RCC project has led to improved outcomes and less acute fatality rates for patients who arrived within this implementation period. However, there were no significant changes in the overall in-hospital mortality [7]. Regarding post-acute care, the RCCs' CR programs are expected to be a model for the Korean CR program. However, an evaluation study on their current status and achievements has not yet been reported. This study's purpose is therefore to evaluate the current status of the CR programs in the RCCs. It also aims to make these data available for developing strategies to boost CR programs in Korea. 


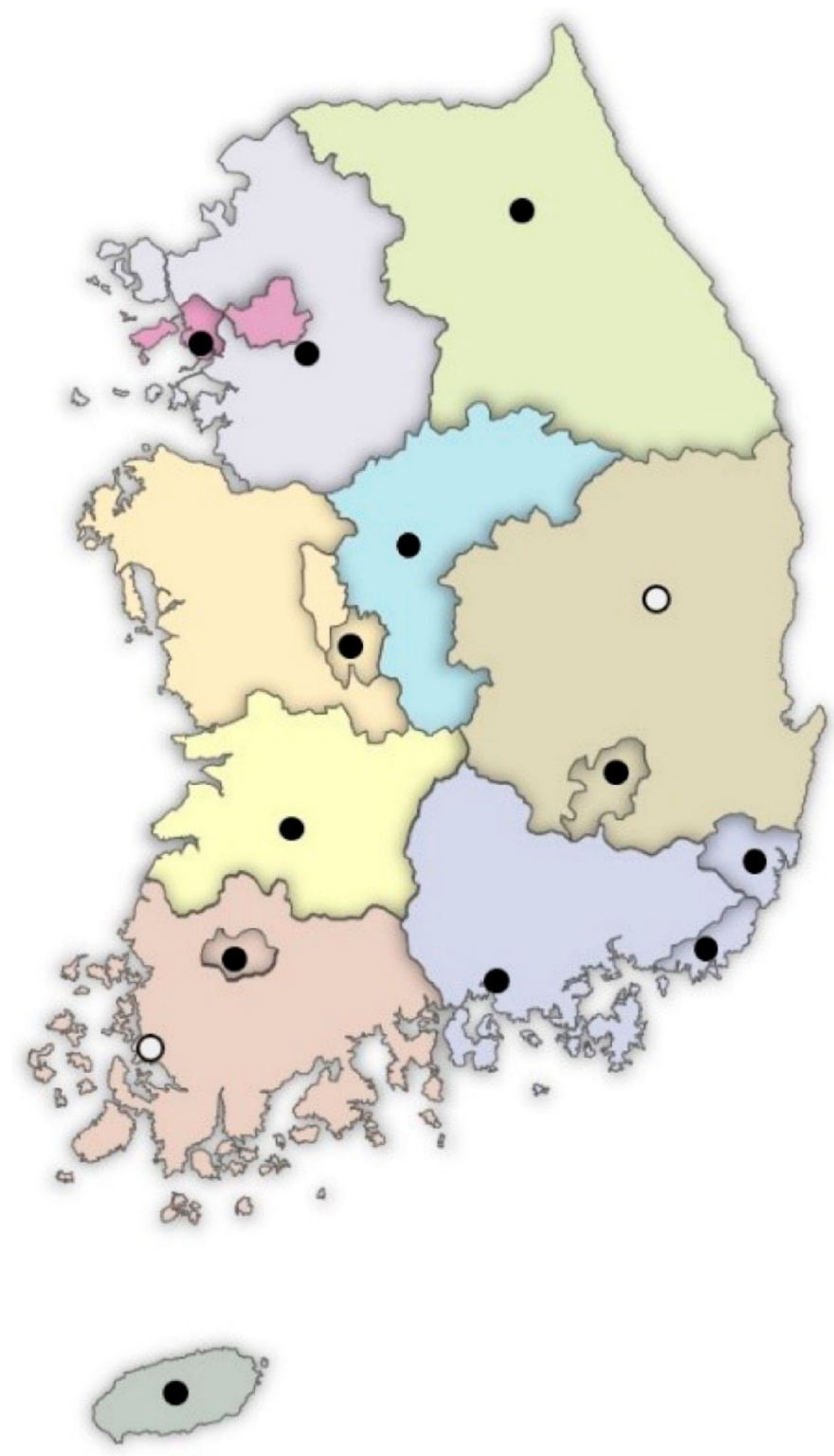

Figure 1. Locations of the Regional Cardiocerebrovascular Centers (RCCs) in South Korea. • Twelve RCCs which are included in this study. $\bigcirc$ Two RCCs which were recently established and are not included in this study.

Table 1. Performance Measure Index for the CR program of RCC (Requirements of the MHW).

\begin{tabular}{|c|c|c|c|}
\hline \multicolumn{2}{|c|}{ Performance Measure Index } & \multirow{2}{*}{$\frac{\text { Definition }}{\text { Documentation of official certification }}$} & \multirow{2}{*}{$\frac{\text { Requirements }}{1}$} \\
\hline \multirow{5}{*}{ Staff } & CR Director & & \\
\hline & Physician & Number of CR specialized physicians & $\geq 1$ \\
\hline & Physical therapist & Number of CR specialized physical therapists & $\geq 1$ \\
\hline & Registered Nurse & Number of CR specialized nurses & $\geq 1$ \\
\hline & ACLS certification & Number of CR staff with ACLS certification & $\geq 1$ \\
\hline \multirow{2}{*}{ Facility } & CV education room & CV education room for patients and families & $\overline{1}$ \\
\hline & Exercise laboratory and gym & Space dedicated for exercise test \& exercise training & Area $\geq 33 \mathrm{~m}^{2}$ \\
\hline \multirow{5}{*}{ Equipment } & Treadmill/Bicycle ergometer & Number of treadmills and bicycle ergometers & $\geq 4$ units \\
\hline & Arm ergometer & Number of arm ergometers & $\geq 1$ unit \\
\hline & CPX test equipment & Number of CPX test equipment & $\geq 1$ unit \\
\hline & ECG telemetry & Number of telemetry ECG channels & $\geq 2$ units \\
\hline & Emergency cart & Number of CPR emergency carts & $\geq 1$ unit \\
\hline Patient data & Patient EMR data sharing & Inter-department patient EMR data sharing & $\overline{\text { Possible }}$ \\
\hline
\end{tabular}


Table 1. Cont.

\begin{tabular}{|c|c|c|c|}
\hline \multicolumn{2}{|c|}{ Performance Measure Index } & Definition & Requirements \\
\hline \multirow{6}{*}{ Operation } & Meeting for CR critical pathway & Regular meeting for practice of CR critical pathways & $\geq 1 /$ month \\
\hline & Update and management of $\mathrm{CP}$ & Update of CR critical pathways and CR protocol & $\geq 1 /$ year \\
\hline & Patient individual education & Offer individual education to CR referred patients & $\geq 80 \%$ \\
\hline & Discharge plan & Offer discharge plan for CR to patients \& families & $100 \%$ \\
\hline & Resnonse to $C R$ referral & Early response to CR referral within $24 \mathrm{~h}$ & $\geq 90 \%$ \\
\hline & kesponse to ck rererral & Offer submaximal exercise test to a CR referred patient & $\geq 50 \%$ \\
\hline \multirow{4}{*}{ Patient CR follow-up } & 3-month CR follow-up & Rate of 3 months CR F/U in CR referred patients & $\geq 50 \%$ \\
\hline & 12-month CR follow-up & Rate of 12 months CR F/U in CR referred patients & $\geq 20 \%$ \\
\hline & 3-month CPX test & Rate of 3 months CPX test in CR referred patients & $\geq 30 \%$ \\
\hline & 6-month CPX test & Rate of 12 months CPX test in CR referred patients & $\geq 20 \%$ \\
\hline \multirow{2}{*}{ Regular meeting } & CR staff meeting & Frequency of regular CR team strategy meeting & $\geq 4 /$ year \\
\hline & National RCC meeting & National annual assembly of RCC staff & Attend \\
\hline
\end{tabular}

CR: cardiac rehabilitation, RCC: regional cardiocerebrovascular center, MHW: Ministry of Health and Welfare, ACLS: advanced cardiac life support, CV: cardiovascular, CPR: cardiopulmonary resuscitation, EMR: electronic medical recording, F/U: follow-up, CPX test: cardiopulmonary exercise test, including respiratory gas analysis.

\section{Materials and Methods}

The study's subjects were the RCCs in South Korea. We excluded the two newly designated RCCs (secondary hospitals) as the CR development processes were still ongoing. The CR program's quality was also unsatisfactory as compared to that of existing RCCs, which are tertiary hospitals. A total of 12 RCCs participated in this study: Chonnam National University Hospital, Chungbuk National University Hospital, Chungnam National University Hospital, Dong-A University Hospital, Gyeongsang National University Hospital, Inha University Hospital, Jeju National University Hospital, Kangwon National University Hospital, Kyungpook National University Hospital, Seoul National University Bundang Hospital, Ulsan University Hospital, and Wonkwang University Hospital.

\subsection{Development of $C R$ Survey Forms}

To examine CR's current status in the 12 RCCs, the CR-General Questionnaire (CRGQ) was developed after analyzing the national and international CR clinical practice guidelines (CPG) [5,8-13]. The Cardiac Rehabilitation-In Depth Questionnaire (CR-IDQ) was developed with reference to the CR evaluation tools of York University $[14,15]$ and International Council of Cardiovascular Prevention and Rehabilitation (ICCPR; www. globalcardiacrehab.com, accessed on 9 September 2021) [16]. To analyze the current status of RCC, CR-GQ examined the: kinds of procedures offered, amount of procedures or surgeries, CR program's initiation, and CR in the institution's delivery system. CR-IDQ investigates the: $C R$ components, $C R$ capacity (patient capacity to be served each year in the institute), CR density, outpatient CR program's commencement time, $C R$ team personnel, facility, supervised exercise program, CPR certification, and further details on the outpatient CR program. Detailed information on CR-GQ and CR-IDQ are available in the Supplementary Materials thru online at https:/ / www.mdpi.com/article/10.3390/jcm1 0215079/s1, Supplementary Materials S1: I. CR-GQ form, Supplementary Materials S2: II. CR-IDQ form.

\subsection{Dispatch of the Surveys}

Printed copies of the CR-GQ were sent to the staff of the cardiology, cardiac surgery, and rehabilitation medicine departments of the 12 RCCs. Printed copies of the CR-IDQ were sent to the staff responsible for CR in the 12 RCCs. The printed surveys, project information, and an explanation of how to respond to the survey were sent in July 2020. An official letter of cooperation from the Korea National Institute of Health was included to increase the response rate. The surveys' responses were entered in a Google survey format that was accessible through a $\mathrm{QR}$ code provided with the package or by connecting to www.crsurvey.co.kr (accessed on 15 September 2020). The data were collected until 30 August 2020. 


\subsection{Confirmation of the Response}

Due to the COVID-19 pandemic, the CR-GQ's response rate was lower than expected. We initially planned to conduct a field survey if the response thereof was inadequate. However, field surveys were not possible as visitors' access to hospitals was restricted due to the pandemic. Out of 44 responses to CR-GQ, the response rate was $59 \%$. This excluded one case of duplicate response and cases with less than $50 \%$ completion. In all 12 RCCs, at least one response was received from the staff of the departments of cardiology and rehabilitation medicine. Responses from the staff of the cardiac surgeries were received from only six centers. All responses to the CR-IDQ were included in the study $(N=12$, response rate 100\%). The staff responsible for CR at each RCC reviewed and confirmed whether the number of candidates and CR participation rates in their RCCs from 1 July 2019 , to 30 June 2020 were correct.

The overall performance achievement was evaluated based on the number of $\mathrm{CR}$ candidates, $C R$ capacity, $C R$ density, and CR participation rate. The number of CR candidates refers to the annual number of AMI admissions less the number of patients not indicated for $\mathrm{CR}$ referral. CR capacity refers to the median number of patients a program can serve annually. CR density refers to the annual number of CR candidates divided by the $C R$ capacity [17]. The CR participation rate refers to the number of outpatient $C R$ enrollments divided by the number of inpatient $C R$ referrals.

\subsection{Statistical Analysis}

To determine whether the CR performance is different among RCCs, we compared the data collected via the surveys from CR directors at the 12 RCCs. An ANOVA was performed to compare $\mathrm{CR}$ candidates, $\mathrm{CR}$ capacity, and CR density. The chi-square test was used to compare the proportion of patients with $\mathrm{CR}$ participation rates. All statistical analyses were performed using the Statistical Package for the Social Sciences (SPSS) (version 19.0; SPSS Inc., Chicago, IL, USA). The level of significance was set at $p<0.05$.

\subsection{Ethics Statement}

This study was approved by the Institutional Review Board of the Inje University Sanggye Paik Hospital, Korea (IRB No. SGPAIK202012010002-HE003). The need to obtain informed consent was waived due to the non-clinical nature of the study. No personal information (patient's name, address, ID, phone number, hospital ID) were collected and thus the participants' anonymity was preserved.

\section{Results}

The following results are based on the responses to CR-GQ and CR-IDQ from the 12 RCCs.

\subsection{Number and Distribution of the CR Programs by Region in Korea}

There were 164 hospitals (103 certified hospitals from the Korean Society of Interventional Cardiology), including the RCCs, which practiced percutaneous coronary intervention (PCI). Out of these 164, $47(28.7 \%)$ had CR programs. There were regional gaps in the CR practice rate when considering the number of ischemic heart disease and AMI cases (Table 2). RCCs were the only hospitals to practice CR in the Daejeon-Chungnam, Chungbuk, Jeonbuk, Ulsan, and Jeju regions.

\subsection{General Characteristics of 12 RCCs Related to the CR Program}

CR programs were established in three RCCs from 2008 to 2009, three RCCs from 2010 to 2011, three RCCs from 2012 to 2013, two RCCs from 2016 to 2017, and three RCCs from 2019 to 2020. There was a total of 14 CR programs in practice. As previously mentioned, we excluded two newly designated RCCs (secondary hospitals) as their CR programs have not yet been established. 
Table 2. Number and Distribution of Hospitals Performing PCI and CR by Region in Korea.

\begin{tabular}{cccccc}
\hline Region ${ }^{\#}$ & RCC & PCI Hospital ${ }^{*}$ & CR Hospital ${ }^{* *}$ & Annul No. of IHD ${ }^{+, \S}$ & Annual No. of AMI ${ }^{\ddagger} \S$ \\
\hline Seoul & 0 & 31 & 13 & 254,753 & 25,715 \\
Gyeonggi & 1 & 33 & 9 & 184,453 & 23,834 \\
Incheon & 1 & 9 & 3 & 48,834 & 5926 \\
Gangwon & 1 & 5 & 2 & 31,748 & 4913 \\
Daejeon-Chungnam & 1 & 12 & 1 & 62,004 & 8204 \\
Chungbuk & 1 & 6 & 1 & 27,432 & 3254 \\
Gwangju-Jeonnam & $2 \mathrm{a}$ & 11 & 2 & 73,777 & 9373 \\
Jeonbuk & 1 & 5 & 1 & 32,372 & 5091 \\
Busan & 1 & 17 & 4 & 84,344 & 8674 \\
Gyeongnam & 1 & 11 & 3 & 51,940 & 7714 \\
Ulsan & 1 & 5 & 1 & 17,988 & 2004 \\
Daegu-Gyeongbuk & $2 \mathrm{~b}$ & 15 & 6 & 93,054 & 15,402 \\
Jeju & 1 & 4 & 1 & 10,563 & 915 \\
Total & 14 & 164 & 47 & 943,006 & 118,872 \\
\hline
\end{tabular}

CR: cardiac rehabilitation, RCC: Regional Cardiocerebrovascular Center. * Province or metropolitan city. * Hospitals that perform PCI (percutaneous coronary intervention). ${ }^{* *}$ Hospitals with CR programs in the region, including RCC. ${ }^{\dagger}$ Ischemic heart disease. ${ }^{\ddagger}$ Acute myocardial infarction. ${ }^{\S}$ Data from Healthcare Big Data Hub, open data on high-interest diseases, 2019 (opendata.hira.or.kr). ${ }^{a}$ 13th RCC was installed in the Jeonnam region in 2020 but was not included in this study. ${ }^{b}$ 14th RCC was installed in the Gyeongbuk region in 2020 but was not included in this study.

In the 12 RCCs, most CVD treatment procedures, such as: PCI, coronary artery bypass graft (CABG) surgery, acute management of heart failure, valvular surgery, percutaneous valve implantation, peripheral arterial stents and surgery, and implantation of pacemakers or implantable cardioverter defibrillators (ICDs), were practiced. However, left ventricular assist device (LVAD) implantation or heart transplants were performed in only seven centers. CR was performed for CABG surgery and angina without stent insertion at seven RCCs. CR was performed for compensated heart failure at six RCCs. CR was performed for valvular surgery, implantation of a pacemaker or ICD, cardiomyopathy, and LVAD at three RCCs. CR was performed for aortic surgery, peripheral artery disease, and heart transplantation at two RCCs.

The annual number of AMI admissions, CR candidates, CR capacity, and CR density in the 12 RCCs are shown in Table 3. The annual number of AMI admissions, CR candidates, CR capacity, and CR density showed significant differences among the 12 RCCs. In particular, the CR capacity and CR density ranged from 50 to 500 and 0.42 to 7.36 , respectively. The RCCs where CR density is more than one need to expand their CR capacity to adequately offer CR programs to each RCC's CR candidate.

\subsection{The CR Program's Current Status in 12 RCCs}

The CR directors at the 12 RCCs were all physiatrists (rehabilitation medicine physicians). At all RCCs, risk factor evaluation, $\mathrm{CR}$ evaluation, $\mathrm{CR}$ exercise prescription, exercise therapy under supervision, drug compliance monitoring, and CR education were conducted. Follow-up assessments were performed at 11 RCCs (92\%). However, psychological evaluation (practice rate 33\%), stress management (practice rate 50\%), and vocational counseling (practice rate $42 \%$ ) were practiced at low rates (Table 4). All RCCs were equipped with the facilities and equipment for CR. However, in some RCCs, general rehabilitation facilities and equipment were used for CR (Table 5). At most centers, the staff held concurrent positions in CR and general rehabilitation. Some personnel were even absent. Therefore, improvements were required (Table 6). At least one member of the CR staff held a license for advanced cardiac life support at all RCCs.

For the CR referral method, nine centers (75\%) used an electronic automatic referral system, while at three centers $(25 \%)$, consultation was made at the prescription of a cardiologist or cardiac surgeon. The CR referral (97\%) and patient education (78\%) rates were high. However, inpatient CR exercise practice $(56 \%)$ was low. There were significant gaps among the 12 RCCs (Figure 2, Table 7). One center did not operate inpatient CR exercise 
practices at all. There were small differences in the time to outpatient CR after PCI by RCCs. However, six RCCs (50\%) began outpatient CR an average of two weeks after PCI. Most RCCs began outpatient CR four weeks after cardiac surgery. However, in five RCCs, outpatient $\mathrm{CR}$ for cardiac surgery patients was not practiced.

At all RCCs, most indicated that patients for CR were AMI patients and those who received PCI. However, this may be because all RCCs are annually evaluated by regular performance measures of KDCA for AMI management. According to the risk stratification for exercise-related adverse $\mathrm{CV}$ complications, high-risk patients participated in outpatient $\mathrm{CR}$ at nine RCCs. Moderate risk patients participated in CR at 11 RCCs, and even low-risk patients participated in CR at eight RCCs.

Table 3. Annual AMI Admission, CR Candidate, CR Capacity, and CR Density in 12 RCCs.

\begin{tabular}{ccccc}
\hline RCC & AMI Admission * $^{*}$ & CR Candidate $^{* *}$ & CR Capacity $^{\dagger}$ & CR Density $^{\ddagger}$ \\
\hline A & 245 & 87 & 70 & 1.24 \\
B & 722 & 589 & 120 & 4.91 \\
C & 471 & 368 & 50 & 7.36 \\
D & 587 & 473 & 100 & 4.73 \\
E & 481 & 487 & 400 & 1.22 \\
F & 163 & 150 & 200 & 0.75 \\
G & 401 & 340 & 220 & 1.55 \\
H & 282 & 210 & 500 & 0.42 \\
I & 1170 & 885 & 300 & 2.95 \\
J & 145 & 131 & 50 & 2.62 \\
K & 362 & 239 & 240 & 0.99 \\
L & 271 & 203 & 300 & 0.80 \\
Total & 5300 & 4162 & 2350 & 1.77 \\
\hline
\end{tabular}

CR: cardiac rehabilitation, RCC: Regional Cardiocerebrovascular Center. * Annual number of acute myocardial infarction admissions for each RCC. ${ }^{* *}$ Annual number of CR candidates for AMI admission of each RCC. ${ }^{\dagger}$ The median number of patients, which each RCC can annually serve. $\ddagger$ CR candidates divided by the CR capacity of each RCC.

Table 4. Offered Cardiac Rehabilitation Components in 12 RCCs.

\begin{tabular}{|c|c|c|c|}
\hline & \multirow[t]{2}{*}{ Components } & \multicolumn{2}{|c|}{$\begin{array}{c}\text { No. of RCCs That Offered a CR } \\
\text { Component That Is Relevant }\end{array}$} \\
\hline & & Yes $(\%)$ & No $(\%)$ \\
\hline \multirow[t]{7}{*}{ Assessments } & Cardiovascular risk factors & $12(100)$ & $0(0)$ \\
\hline & Cardiopulmonary exercise test & $12(100)$ & $0(0)$ \\
\hline & Other physical function test & $10(83)$ & $2(7)$ \\
\hline & Assessment of comorbidity & $9(83)$ & $3(27)$ \\
\hline & Psychological evaluation & $4(33)$ & $8(67)$ \\
\hline & Follow-up after the end of program & $11(92)$ & $1(8)$ \\
\hline & Long-term follow-up & $11(92)$ & $1(8)$ \\
\hline \multirow[t]{4}{*}{ Management } & Exercise prescription & $12(100)$ & $0(0)$ \\
\hline & Supervised exercise training & $12(100)$ & $0(0)$ \\
\hline & Self-monitoring technique & $12(100)$ & $0(0)$ \\
\hline & Stress management & $6(50)$ & $6(50)$ \\
\hline \multirow[t]{4}{*}{ Education } & Risk factors control & $12(100)$ & $0(0)$ \\
\hline & CV drug compliance & $12(100)$ & $0(0)$ \\
\hline & Nutritional counseling & $9(83)$ & $3(27)$ \\
\hline & Vocational counseling & $5(42)$ & $7(58)$ \\
\hline
\end{tabular}

RCC: Regional Cardiocerebrovascular Center. CV: Cardiovascular. 
Table 5. Facility and Equipment for Cardiac Rehabilitation Program in 12 RCCs.

\begin{tabular}{cccc}
\hline \multirow{2}{*}{ Facility or Equipment } & \multicolumn{3}{c}{ No. of RCCs Holding Facility or Equipment } \\
\cline { 2 - 4 } & Exclusive (\%) & Concurrent (\%) & Absence (\%) \\
\hline Exercise Gym & $12(100)$ & $0(0)$ & $0(0)$ \\
Treadmill & $12(100)$ & $0(0)$ & $0(0)$ \\
Bicycle ergometer & $11(92)$ & $1(8)$ & $0(0)$ \\
Arm ergometer & $5(42)$ & $5(42)$ & $2(17)$ \\
CPX test equipment & $12(100)$ & $0(0)$ & $0(0)$ \\
Telemetry ECG monitoring system & $12(100)$ & $0(0)$ & $0(0)$ \\
Oxygen supply & $12(100)$ & $0(0)$ & $0(0)$ \\
Education room & $10(83)$ & $2(17)$ & $0(0)$ \\
Locker room & $8(67)$ & $4(33)$ & $0(0)$ \\
Resistance training equipment & $8(67)$ & $4(33)$ & $0(0)$ \\
Body composition analyzer & $8(67)$ & $2(17)$ & $2(17)$ \\
\hline
\end{tabular}

RCCs: Regional Cardiocerebrovascular Center. CPX test: cardiopulmonary exercise test. ECG: electrocardiography.

Table 6. Staff Composition for Cardiac Rehabilitation Program in 12 RCCs.

\begin{tabular}{cccc}
\hline \multirow{2}{*}{ Staff Composition } & \multicolumn{2}{c}{ No. of RCCs Assigned Staff Composition } \\
\cline { 2 - 4 } & Exclusive (\%) & Concurrent (\%) & Absence (\%) \\
\hline Director & $0(0)$ & $12(100)$ & $0(0)$ \\
Physical therapist & $10(83)$ & $2(17)$ & $0(0)$ \\
Nurse & $6(50)$ & $6(50)$ & $0(0)$ \\
Psychologist & $0(0)$ & $4(33)$ & $8(67)$ \\
Nutritionist & $2(17)$ & $5(42)$ & $5(42)$ \\
\hline
\end{tabular}

RCCs: Regional Cardiocerebrovascular Center.

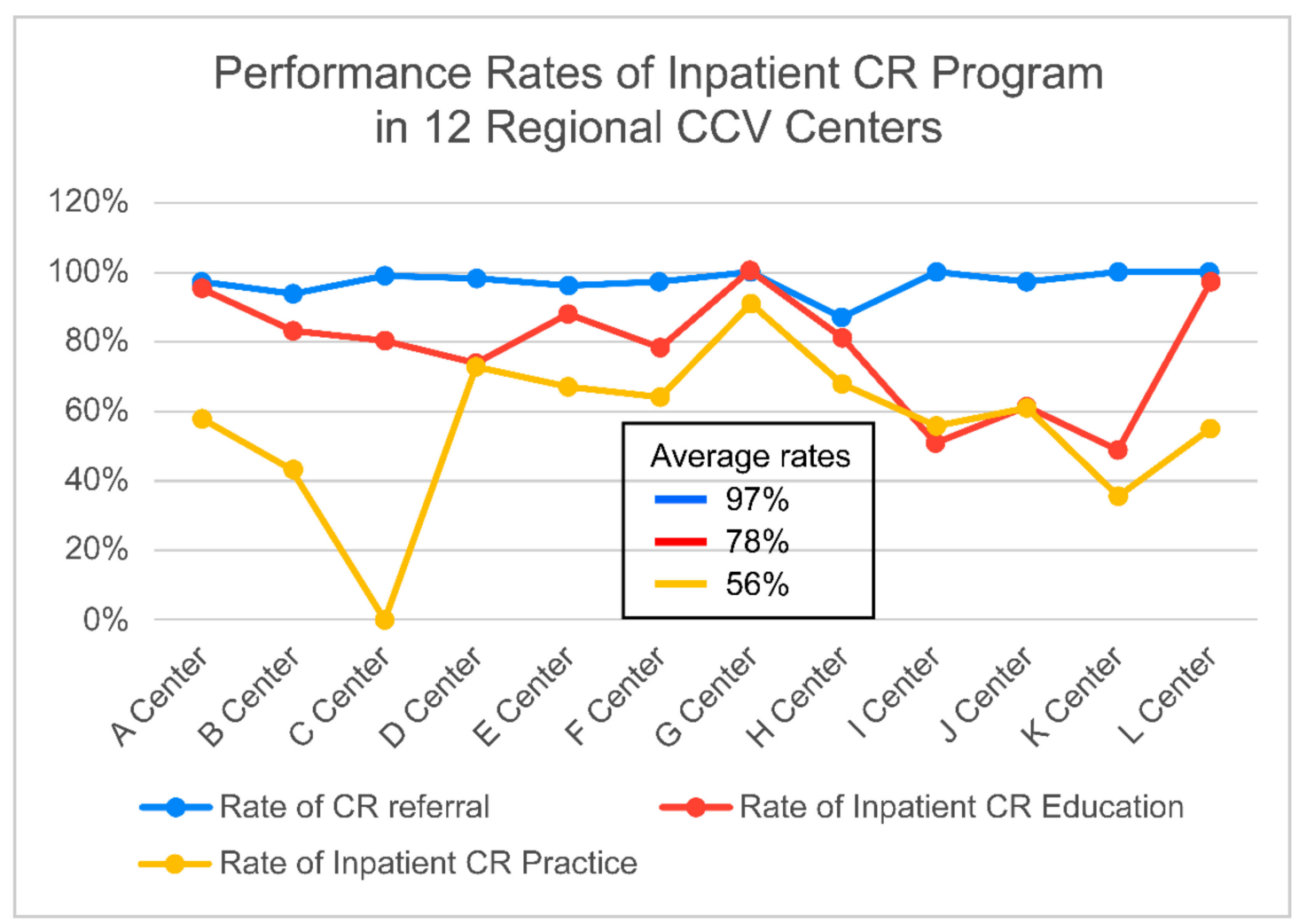

Figure 2. Performance Rate of Inpatient CR Program in 12 RCCs. CR: cardiac rehabilitation, RCC: Regional Cardiocerebrovascular Center. 
Table 7. Rate of CR Referral, Patient Education, and Inpatient CR Program in Each RCC.

\begin{tabular}{cccc}
\hline RCC & CR Referral & Patient Education & Inpatient CR Program $^{+}$ \\
\hline A & $97 \%$ & $95 \%$ & $58 \%$ \\
B & $94 \%$ & $83 \%$ & $43 \%$ \\
C & $99 \%$ & $80 \%$ & $0 \%$ \\
D & $98 \%$ & $74 \%$ & $73 \%$ \\
E & $96 \%$ & $88 \%$ & $67 \%$ \\
F & $97 \%$ & $78 \%$ & $64 \%$ \\
G & $100 \%$ & $100 \%$ & $91 \%$ \\
H & $87 \%$ & $81 \%$ & $68 \%$ \\
I & $100 \%$ & $51 \%$ & $56 \%$ \\
J & $97 \%$ & $61 \%$ & $61 \%$ \\
K & $100 \%$ & $49 \%$ & $36 \%$ \\
L & $100 \%$ & $97 \%$ & $55 \%$ \\
Total & $97 \%$ & $78 \%$ & $56 \%$ \\
\hline
\end{tabular}

CR: cardiac rehabilitation, RCC: Regional Cardiocerebrovascular Center. ${ }^{*}$ Rate of CR referral $=$ no. of CR referrals divided by the no. of the annual CR candidates for AMI admission. ** The rate of patient education $=$ no. of patient education divided by the annual no. for CR referral for AMI admission. + Rate of inpatient $\mathrm{CR}$ program $=$ no. of the inpatient CR program divided by annual no. for CR referral for AMI admission.

The enrollment $(47 \%)$ and adherence $(17 \%)$ rates to the outpatient CR program were much lower and showed deviations among RCCs (Figure 3). Full planned session of formal out-patient CR program are 36 sessions (three times a week for 3 months). The rates of each RCC are listed in Table 8. On a Likert scale ranging from one to five, the opinions of medical staff in 12 RCCs on outpatient CR barriers were 4.1 for time/distance/transport issues. They were 3.5 for patients' burden of CR expense, and 3.0 for a lack of recommendation to attend an outpatient $\mathrm{CR}$ program by a cardiologist and cardiac surgeon and a lack of $\mathrm{CR}$ staff (Table 9).

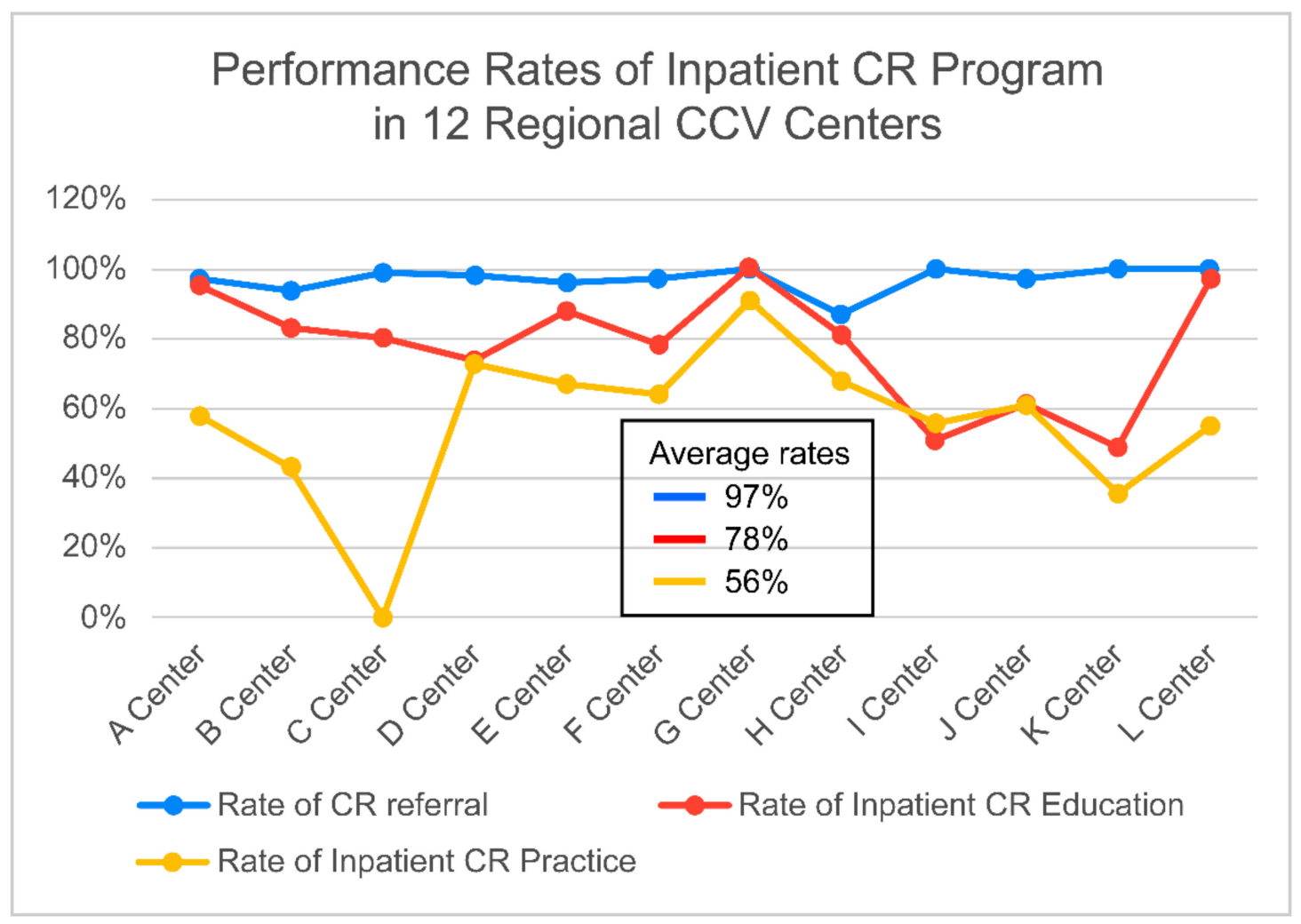

Figure 3. Performance Rate of Outpatient CR Program in 12 RCCs. CR: cardiac rehabilitation, RCC: Regional Cardiocerebrovascular Center. 
Table 8. Rate of Outpatient CR Enrollment, Performing Baseline CPX Test, and CR Adherence.

\begin{tabular}{cccc}
\hline RCC & CR Enrollment & Baseline CPX Test & CR Adherence \\
\hline A & $37 \%$ & $20 \%$ & $12 \%$ \\
B & $30 \%$ & $28 \%$ & $30 \%$ \\
C & $34 \%$ & $33 \%$ & $3 \%$ \\
D & $44 \%$ & $44 \%$ & $18 \%$ \\
E & $31 \%$ & $19 \%$ & $14 \%$ \\
F & $77 \%$ & $34 \%$ & $1 \%$ \\
G & $44 \%$ & $48 \%$ & $6 \%$ \\
H & $67 \%$ & $53 \%$ & $11 \%$ \\
I & $48 \%$ & $29 \%$ & $26 \%$ \\
J & $43 \%$ & $54 \%$ & $31 \%$ \\
K & $57 \%$ & $27 \%$ & $31 \%$ \\
L & $51 \%$ & $44 \%$ & $19 \%$ \\
Total & $47 \%$ & $36 \%$ & $17 \%$ \\
\hline
\end{tabular}

CR: cardiac rehabilitation, CPX test: cardiopulmonary exercise test. Rate of CR enrollment $=$ no. of outpatient $\mathrm{CR}$ enrollment divided by the no. for CR referral during admission. Rate of baseline CPX test $=$ no. of PCX test completed/no. of CR referral. Rate of CR adherence $=$ no. of outpatient CR Completion/no. of CR referral. RCC: Regional Cardiocerebrovascular Center.

Table 9. Likert Scales of the Physician's Opinion on Outpatient CR Barriers in 12 RCCs.

\begin{tabular}{ccccccc}
\hline RCC & $\begin{array}{c}\text { Lack of } \\
\text { Recommendation * }\end{array}$ & $\begin{array}{c}\text { Lack of CR } \\
\text { Equipment }\end{array}$ & $\begin{array}{c}\text { Lack of } \\
\text { CR Space }\end{array}$ & $\begin{array}{c}\text { Lack of } \\
\text { CR Staff }\end{array}$ & $\begin{array}{c}\text { Pt's Burden } \\
\text { of Payment }\end{array}$ & $\begin{array}{c}\text { Time/Distance/ } \\
\text { Transport Issues ** }\end{array}$ \\
\hline A & $3.0 \pm 0.81$ & $1.7 \pm 0.47$ & $1.7 \pm 0.47$ & $2.0 \pm 0.81$ & $3.3 \pm 0.47$ & $4.3 \pm 0.47$ \\
B & $3.5 \pm 0.50$ & $3.5 \pm 0.50$ & $3.5 \pm 0.50$ & $4.0 \pm 0.00$ & $3.5 \pm 0.50$ & $4.5 \pm 0.50$ \\
C & $4.0 \pm 0.70$ & $2.0 \pm 1.00$ & $2.0 \pm 1.00$ & $2.5 \pm 1.10$ & $4.5 \pm 0.82$ & $4.5 \pm 0.86$ \\
D & $2.5 \pm 0.50$ & $1.5 \pm 0.50$ & $1.0 \pm 0.00$ & $4.5 \pm 0.50$ & $4.5 \pm 0.50$ & $4.5 \pm 0.50$ \\
E & $1.5 \pm 0.50$ & $1.0 \pm 0.00$ & $2.0 \pm 1.00$ & $2.0 \pm 1.00$ & $2.5 \pm 0.50$ & $4.0 \pm 0.00$ \\
F & $3.0 \pm 0.81$ & $3.3 \pm 0.47$ & $3.4 \pm 0.47$ & $3.7 \pm 0.47$ & $3.0 \pm 0.00$ & $3.3 \pm 1.24$ \\
G & $3.0 \pm 1.00$ & $1.5 \pm 0.50$ & $1.5 \pm 0.50$ & $2.0 \pm 1.00$ & $3.5 \pm 0.50$ & $4.0 \pm 1.00$ \\
H & $4.0 \pm 0.00$ & $1.5 \pm 0.50$ & $1.5 \pm 0.50$ & $1.5 \pm 0.50$ & $3.5 \pm 0.00$ & $4.0 \pm 0.00$ \\
I & $3.0 \pm 1.26$ & $2.0 \pm 0.89$ & $2.4 \pm 0.80$ & $3.2 \pm 0.40$ & $3.4 \pm 0.48$ & $4.0 \pm 0.89$ \\
J & $2.5 \pm 1.50$ & $1.3 \pm 0.43$ & $1.8 \pm 0.82$ & $2.5 \pm 1.00$ & $3.5 \pm 0.50$ & $3.8 \pm 0.43$ \\
K & $4.3 \pm 0.82$ & $2.3 \pm 1.29$ & $2.5 \pm 1.11$ & $3.8 \pm 0.43$ & $3.5 \pm 0.50$ & $4.0 \pm 0.70$ \\
L & $3.0 \pm 1.00$ & $1.5 \pm 0.50$ & $1.5 \pm 0.50$ & $3.0 \pm 2.00$ & $3.5 \pm 0.50$ & $4.0 \pm 0.00$ \\
Total & $3.1 \pm 1.21$ & $2.2 \pm 1.05$ & $2.2 \pm 1.12$ & $2.9 \pm 1.23$ & $3.5 \pm 0.72$ & $4.0 \pm 0.82$ \\
\hline
\end{tabular}

Values are mean \pm standard deviation, Likert scale: 1—Definitely not (strongly disagree), 2-Not (disagree), 3-Neutral (neutral), 4-Yes, but minor (agree), 5-Major issue (strongly agree). CR: cardiac rehabilitation, RCC: Regional Cardiocerebrovascular Center. ${ }^{*}$ Lack of recommendation to attend the outpatient CR program by a cardiologist or cardiac surgeon. ${ }^{* *}$ Time, distance, or transport issues to visit a hospital for the outpatient CR program.

\section{Discussion}

CR is practiced in 111 countries globally [17], and countries including the US, Canada, Europe, Japan, and Korea have published high-quality CPG [5,8-13]. Although the level of recommendation for CR is strong, the actual CR participation rate is only about $30-40 \%$ [18]. Each nation is striving to increase the participation rate. However, this rate is only about $1.5 \%$ in Korea [19], which is much lower than those of the other aforementioned countries. In addition, there are a lack of strategies for effective management in the community after patients' discharge and for increasing treatment compliance. The continuum of care from the hospital to the community after patient discharge is very important for determining their long-term prognosis. Therefore, a state-led system needs to be established.

A comprehensive CR program was introduced in Korea around the late 1990s. Before the establishment of RCCs, only five private medical institutions had well-organized CR programs in South Korea. They were all in densely populated areas in the capital region. Before the regional hospitals in this study were granted RCC status by the MHW, they had no CR facility, equipment, or CR specialized staff. Since 2008, the MHW of South Korea has established 14 RCCs in different provinces nationwide. This includes 12 tertiary 
university hospitals and two general hospitals. The intention was to reduce the medical gaps in research, knowledge, and practice for AMIs and strokes among the regions in South Korea. There may be differences in the organization of nationwide infrastructure for the CV care systems in other countries. However, it is expected that RCCs serve as key units of organization to provide nationwide quality care for CVD in South Korea. Standardized, high-quality care for AMI and stroke has been established in the RCCs. Furthermore, equipment and maintenance of CR facilities and personnel are mandatory. Many of this study's co-authors have published articles on CR's status and impact on AMI's prognosis [20-24]. There has been an increasing acceptance of CR nationwide and an introduction of CR insurance benefits in February 2017. CR facilities have therefore increased by up to 46 centers nationwide. This includes RCCs. A growing number of medical institutions are preparing to establish CR programs. However, the CR participation rate is still very low and there are considerable differences in the CR programs' activity levels among CR facilities [19].

According to this study's results, considering the number of CVD patients by region, the CR demand cannot be met by CR programs in the RCCs. In Daejeon-Chungnam, Chungbuk, Jeonbuk, Ulsan, and Jeju regions, RCCs were the only hospitals practicing CR. The history of CR programs at the RCCs ranges from 2 to 13 years. Regardless of the duration, the number of AMI patients, physicians' interest in and will to practice CR, and regional socioeconomic characteristics contributed to the CR programs' activity levels in each RCC. The CR capacity ranged from 50 to 500. The CR density varied from 0.42 to 7.36. RCCs with a CR density of more than one, due to low CR capacity, need to expand their CR capacity to sufficiently offer CR programs to their candidates. In particular, B, $C$, and D centers should endeavor to urgently improve their CR capacity to reserve their standardized patient quota.

At most RCCs, the facilities, equipment, and personnel required for CR were in place. However, there were some RCCs that concurrently utilized general rehabilitation facilities and personnel for CR. This practice should be reformed. At all RCCs, risk factor evaluation, CR evaluation, CR exercise prescription, exercise therapy under supervision, drug compliance monitoring, and CR education were performed. However, psychological evaluation, stress management, and vocational counseling were practiced at low rates. These components must also be added to the comprehensive CR program for all RCCs [25-27].

The average RCCs' CR referral rate was $97 \%$. This is very high, which may be because nine RCCs (75\%) used automatic referral systems. Furthermore, the medical staff reviewed the prescription for CR referral at three RCCs (25\%). However, as compared to the inpatient $C R$ referral rate, the outpatient $C R$ participation rate was very low. The enrollment rate in (47\%) and adherence to (17\%) outpatient CR were much lower and showed deviations among the 12 RCCs. We are currently in the process of analyzing big data using health insurance claim data showing implementation status of CR in Korea including CR participation rate.

The factors that hinder participation may be a lack of understanding of the need for CR, lack of motivation, and various socioeconomic factors. These include time/distance/transport issues and the burden of paying CR fees. As compared to tertiary medical centers in Seoul and the metropolitan areas, the proportion of patients coming from farming/fishing villages, mountainous villages, and islands is relatively high at the RCCs. It is therefore difficult to maintain adherence to hospital-based outpatient CR. Grace et al. and a study by four institutions in Korea have reported similar barriers to CR [28,29].

Strategies are required to overcome these barriers at the patient, doctor, hospital, and policy levels $[30,31]$. According to this study's results, the high average rate of CR referral $(97 \%)$ is satisfactory. However, the low rate of CR enrollment (47\%) and adherence (17\%) to the outpatient $\mathrm{CR}$ programs are major challenges to be overcome regarding patientcentered strategies. First, to improve CR facilities' accessibility, CR programs need to be established in more hospitals to form a denser CR network. According to a report on the global CR density status in 2019, the CR density in South Korea was 22. It ranked 27th out 
of 111 countries with CR programs [17]. Thus, RCCs should be expanded for improving the $\mathrm{CR}$ capacity to meet the need in their region and a regional network system should be implemented through the new "Local Cardiocerebrovascular Center (LCC)" project. This is currently being developed by the government. It not only provides timely acute medical services but also provides post discharge outcome care across the country, including rural areas [32]. For this second mission, CR programs' installation should be made mandatory in all LCCs. The experience of CR programs in RCCs may play a central role in the establishment of CR programs in LCCs. RCCs may serve as leaders of CR networks in each region (CR hub). This may provide education and support for the LCCs and establish a CR network, where the centers may refer CR patients mutually. More flexible scheduling for outpatient $C R$, improvement in accessibility to CR facilities (location and direction signs) in hospitals, and more active utilization of home-based CR programs should be applied [33,34]. These include the development of standardized protocols and insurance fees, game type $\mathrm{CR}$ programs, and telehealth or mobile healthcare techniques. We are currently conducting a randomized controlled trials that applies hybrid CR and telerehabilitation, the strategies for improvement of $C R$ participation, as subsequent research.

At a patient level, RCCs should provide self-efficacy counseling and education programs, psychological evaluation, and consultation to improve patients' motivation. They could also provide specialized CR programs for elderly patients with severely reduced exercise capacity, transportation/mobility aid for the elderly, and CR participation incentives. These include reimbursement for transportation expenses or gifts for successful completion of the CR program. In addition, a CR co-pay relief program should be implemented to reduce the patients' $C R$ fee burden.

This study has several limitations that should be noted. First, it was based on questionnaire surveys. Since they were completed by at least one cardiologist, cardiac surgeon, or physiatrist, there is a possibility of response bias. Second, the COVID-19 pandemic adversely impacted the study's response rate from the eligible sample. Third, since the CR in-depth questionnaires were completed by only a physiatrist, the surveys' results may not reflect the cardiologists' and cardiac surgeons' perspectives. This was because the CR directors of all the RCCs were physiatrists. Future studies should include hospitals where $\mathrm{CR}$ directors are cardiologists or cardiac surgeons.

Despite these limitations, this study is meaningful, as it is the first study to examine and compare CR status in government-sponsored RCCs. The results showed that while RCCs are equipped with the necessary facilities, equipment, and personnel, not all the essential components are in place. More standardized clinical performance and quality measures should be used to improve the CR quality in RCCs $[20,21]$. The outpatient CR program is still not as active as the inpatient program. There are substantial gaps among the RCCs. Therefore, to improve CR in the RCCs, the attention and resources of the medical staff, hospital management, and standardization of the CR programs in the RCCs are required. In addition, patient-oriented $\mathrm{CR}$ programs should be more actively practiced to increase the rate of outpatient $\mathrm{CR}$ adherence. However, for the aforementioned strategies to be realized, effective governmental policy and financial support are required.

CR has been reimbursed by the Korean government since February 2017. There has yet to be a national systematic plan for improving CR implementation, and a state-controlled CR network has not yet been established as well. The activation of CR in Korea was somewhat delayed compared to other high-income countries. To overcome this situation, 12 government-driven RCCs to cover CR were established across the whole country. There are many countries where the status of CR is similar to Korea, or not yet activated. We believe it would be beneficial for such countries to refer to the situation in Korea.

Through three consecutive years of research, we intend to present strategies to improve $\mathrm{CR}$ participation in Korea. As the first step of this project, we investigated the current status of CR in Korea through a nationwide survey. This article, a part of the complete work, details the operating status of 12 RCCs established with government support. 


\section{Conclusions}

$\mathrm{CR}$ in Korea began approximately 30 years after most medically advanced countries. However, the Korean KDCA realized CR's importance and mandated the CR programs' implementation in the RCC project. According to this study's results, while the CR programs are in operation at an elementary level, there are improvements required. There is a large gap among the RCCs. Therefore, specific and active standardization procedures are necessary. A patient-oriented approach should be actively practiced. When appropriate improvements and financial/political support are made, the CR programs of the RCCs will be able to reach global standards.

Supplementary Materials: The following are available online at https:/ /www.mdpi.com/article/10 .3390/jcm10215079/s1, Supplementary Material S1: I. CR-GQ form, Supplementary Material S2: II. CR-IDQ form.

Author Contributions: Conceptualization, C.K., J.S., J.-Y.H., S.J., J.W.L., J.H.L., W.-S.K., H.J.B., S.B., K.-L.J., A.R.K., S.Y.L., J.K., C.R.K. and O.P.K.; methodology, C.K., J.S., J.-Y.H., S.J. and J.W.L.; formal analysis, C.K., J.S., J.-Y.H., S.J. and J.W.L.; data curation, C.K., J.S., J.-Y.H., S.J., J.W.L., J.H.L., W.-S.K., H.J.B., S.B., K.-L.J., A.R.K., S.Y.L., J.K., C.R.K. and O.P.K.; writing-original draft preparation, C.K.; writing-review and editing, J.S., J.-Y.H., S.J. and J.W.L.; project administration, C.K.; funding acquisition, C.K. All authors have read and agreed to the published version of the manuscript.

Funding: This research was funded by the Research Program, which was funded by the Korea National Institute of Health (2020-ER6305-01).

Institutional Review Board Statement: The study was conducted according to the guidelines of the Declaration of Helsinki, and approved by the Institutional Review Board (or Ethics Committee) of INJE UNIVERSITY SANGGYE PAIK HOSPITAL (IRB No. SGPAIK202004013; 29 April 2020).

Informed Consent Statement: Subject consent was waived due to the non-clinical nature of this study.

Data Availability Statement: The data that support the findings of this study are available from the Korea National Institute of Health (KNIH) but restrictions apply to the availability of these data, which were used under license for the current study, and so are not publicly available. Data are however available from the authors upon reasonable request and with permission of KNIH.

Acknowledgments: The authors express their gratitude to Sherry L Grace, York University, and ICCPR, who inspired us to investigate the status of CR and shared the questionnaire forms with us. We are grateful to Jeong Rim Moon, a physiatrist and former member of the National Assembly, and Kun-Sei Lee, Department of Preventive Medicine, School of Medicine, Konkuk University, for their efforts in developing and supporting the RCC project.

Conflicts of Interest: The authors declare no conflict of interest. The funders had no role in the design of the study; in the collection, analyses, or interpretation of data; in the writing of the manuscript, or in the decision to publish the results.

\section{References}

1. Kim, H.; Kim, S.; Han, S.; Rane, P.P.; Fox, K.M.; Qian, Y.; Suh, H.S. Prevalence and incidence of atherosclerotic cardiovascular disease and its risk factors in Korea: A nationwide population-based study. BMC Public Health 2019, 19, 1112. [CrossRef]

2. Lee, S.W.; Kim, H.C.; Lee, H.S.; Suh, I. Thirty-year trends in mortality from cardiovascular diseases in Korea. Korean Circ. J. 2015, 45, 202-209. [CrossRef] [PubMed]

3. Cause of Death Statistics. Korean Statistical Information Service. Available online: https://kostat.go.kr/portal/korea/kor_nw/ 1/6/2/index.board?bmode=read\&aSeq=385219 (accessed on 9 September 2021).

4. OECD. OECD Reviews of Health Care Quality: Korea 2012: Raising Standards. In OECD Reviews of Health Care Quality; OECD: Paris, France, 2012. [CrossRef]

5. Lewanczuk, R.; Suskin, N.; Arthur, H.M. Principles of Chronic Disease Care. In Canadian Guidelines for Cardiac Rehabilitation and Cardiovascular Disease Prevention: Translating Knowledge into Action, 3rd ed.; Canadian Association of Cardiac Rehabilitation: Winnipeg, MB, Canada, 2009; pp. 63-68.

6. Lee, K.S. The Role and Designation Criteria of Cardio-Cerebrovascular Center; Ministry of Health and Welfare: Sejong, Korea, 2018.

7. Kim, A.; Yoon, S.J.; Kim, Y.A.; Kim, E.J. The burden of acute myocardial infarction after a regional cardiovascular center project in Korea. Int. J. Qual. Health Care 2015, 27, 349-355. [CrossRef] 
8. Scottish Intercollegiate Guidelines Network (SIGN). Cardiac Rehabilitation: A National Clinical Guideline. Available online: https:/ /www.sign.ac.uk/our-guidelines/sign-57-cardiac-rehabilitation/ (accessed on 9 September 2021).

9. Adams, P.; Bennet, I.; Carver, K.; Cunningham, W.; Jones, J.; Levie, C.; Murphy, J.; Ramdany, S.; Speck, L.; Wright, P. Myocardial Infarction: Cardiac Rehabilitation and Prevention of Further Cardiovascular Disease (CG172). Available online: https: / /www. nice.org.uk/guidance/cg172 (accessed on 9 September 2021).

10. Smith, S.C., Jr.; Benjamin, E.J.; Bonow, R.O.; Braun, L.T.; Creager, M.A.; Franklin, B.A.; Gibbons, R.J.; Grundy, S.M.; Hiratzka, L.F.; Jones, D.W.; et al. AHA/ACCF secondary prevention and risk reduction therapy for patients with coronary and other atherosclerotic vascular disease: 2011 update: A guideline from the American Heart Association and American College of Cardiology Foundation endorsed by the World Heart Federation and the Preventive Cardiovascular Nurses Association. Circulation 2011, 124, 2458-2473. [PubMed]

11. Piepoli, M.F.; Hoes, A.W.; Agewall, S.; Albus, C.; Brotons, C.; Catapano, A.L.; Cooney, M.T.; Corrà, U.; Cosyns, B.; Deaton, C.; et al. 2016 European Guidelines on cardiovascular disease prevention in clinical practice: The Sixth Joint Task Force of the European Society of Cardiology and Other Societies on Cardiovascular Disease Prevention in Clinical Practice (constituted by representatives of 10 societies and by invited experts) Developed with the special contribution of the European Association for Cardiovascular Prevention \& Rehabilitation (EACPR). Eur. Heart J. 2016, 37, 2315-2381.

12. JCS Joint Working Group. Guidelines for rehabilitation in patients with cardiovascular disease (JCS 2012). Circ. J. 2014, 78, 2022-2093. [CrossRef] [PubMed]

13. Kim, C.; Sung, J.; Lee, J.H.; Kim, W.S.; Lee, G.J.; Jee, S.; Jung, I.Y.; Rah, U.W.; Kim, B.O.; Choi, K.H.; et al. Clinical practice guideline for cardiac rehabilitation in Korea. Ann. Rehabil. Med. 2019, 43, 355-443. [CrossRef] [PubMed]

14. Cardiac Rehabilitation Referral Tools. Available online: https://sgrace.info.yorku.ca/cardiac-rehabilitation-referral-tools/ (accessed on 9 September 2021).

15. Cardiac Rehabilitation Barriers Scales. Available online: https://journals.sagepub.com/doi/pdf/10.1177/0269215511410579 (accessed on 9 September 2021).

16. International Council of Cardiovascular Prevention and Rehabilitation. Global Survey of Cardiac Rehabilitation. Available online: https:/ / www.thelancet.com/journals/eclinm/article/PIIS2589-5370(19)30099-9/fulltext (accessed on 9 September 2021).

17. Turk-Adawi, K.; Supervia, M.; Lopez-Jimenez, F.; Pesah, E.; Ding, R.; Britto, R.R.; Bjarnason-Wehrens, B.; Derman, W.; Abreu, A.; Babu, A.S.; et al. Cardiac rehabilitation availability and density around the globe. EClinicalMedicine 2019, 13, 31-45. [CrossRef] [PubMed]

18. Goto, Y. Current state of cardiac rehabilitation in Japan. Prog. Cardiovasc. Dis. 2014, 56, 557-562. [CrossRef] [PubMed]

19. Kim, S.H.; Ro, J.; Leigh, J.; Kim, W. Underutilization of hospital-based cardiac rehabilitation after acute myocardial infarction in Korea. J. Korean Med. Sci. 2020, 35, e262. [CrossRef]

20. Kim, C.; Choi, I.; Cho, S.; Han, J.Y.; Kim, A.R.; Kim, W.S.; Jee, S.; Lee, J.H.; Joo, M.C.; Bang, H.J.; et al. Cardiac rehabilitation and 5-year mortality after acute myocardial infarction. Report from 11 tertiary hospitals in Korea (ETHIK Study). Eur. J. Phys. Rehabil. Med. 2020, 56, 489-495. [CrossRef]

21. Seo, Y.G.; Jang, M.J.; Park, W.H.; Hong, K.P.; Sung, J. Inpatient cardiac rehabilitation programs' exercise therapy for patients undergoing cardiac surgery: National Korean Questionnaire Survey. J. Exerc. Rehabil. 2017, 13, 76-83. [CrossRef] [PubMed]

22. Kim, C.; Choi, I.; Cho, S.; Kim, A.R.; Kim, W.; Jee, S. Do cardiac rehabilitation affect clinical prognoses such as recurrence, readmission, revascularization, and mortality after AMI? Systematic review and meta-analysis. Ann. Rehabil. Med. 2021, 45, 57-70. [CrossRef] [PubMed]

23. Baek, S.; Ha, Y.; Mok, J.; Park, H.W.; Son, H.R.; Jin, M.S. Community-based cardiac rehabilitation conducted in a public health center in South Korea: A preliminary study. Ann. Rehabil. Med. 2020, 44, 481-492. [CrossRef]

24. Supervia, M.; Turk-Adawi, K.; Lopez-Jimenez, F.; Pesah, E.; Ding, R.; Britto, R.R.; Bjarnason-Wehrens, B.; Derman, W.; Abreu, A.; Babu, A.S.; et al. Nature of cardiac rehabilitation around the globe. EClinicalMedicine 2019, 13, 46-56. [CrossRef]

25. Writing Committee Members; Thomas, R.J.; King, M.; Lui, K.; Oldridge, N.; Piña, I.L.; Spertus, J. AACVPR/ACCF / AHA 2010 update: Performance measures on cardiac rehabilitation for referral to cardiac rehabilitation/secondary prevention services: A report of the American Association of Cardiovascular and Pulmonary Rehabilitation and the American College of Cardiology Foundation/American Heart Association Task Force on Performance Measures (Writing Committee to Develop Clinical Performance Measures for Cardiac Rehabilitation). Circulation 2010, 122, 1342-1350. [PubMed]

26. Thomas, R.J.; Balady, G.; Banka, R.; Beckie, T.M.; Chiu, J.; Gokak, S.; Ho, P.M.; Keteyian, S.J.; King, M.; Lui, K.; et al. 2018 ACC/AHA Clinical performance and quality measures for cardiac rehabilitation: A report of the American College of Cardiology/American Heart Association Task Force on Performance Measures (Developed in collaboration with the American Association of Cardiovascular and Pulmonary Rehabilitation). J. Am. Coll. Cardiol. JACC 2018, 71, $1814-1837$.

27. Kim, C.; Bang, H.J.; Kim, J.H.; Sohn, M.K.; Yang, C.Y.; Lee, S.G.; Lee, E.S.; Lee, J.H.; Im, S.H.; Jung, T.D.; et al. Recommendations for establishing cardiac rehabilitation programs; Facility, equipment and staff: The Korean Society of Cardiac Rehabilitation (KSCR) position statement. J. Korean Acad. Rehabil. Med. 2010, 34, 491-497.

28. Grace, S.L.; Shanmugasegaram, M.S.; Gravely-Witte, M.S.; Brual, M.J.; Suskin, N.; Stewart, D.E. Barriers to cardiac rehabilitation: Does age make a difference? J. Cardiopulm. Rehabil. Prev. 2009, 29, 183-187. [CrossRef]

29. Im, H.W.; Baek, S.; Jee, S.; Ahn, J.M.; Park, M.W.; Kim, W.S. Barriers to outpatient hospital-based cardiac rehabilitation in Korean patients with acute coronary syndrome. Ann. Rehabil. Med. 2018, 42, 154-165. [CrossRef] 
30. Balady, G.J.; Ades, P.A.; Bittner, V.A.; Franklin, B.A.; Gordon, N.F.; Thomas, R.J.; Tomaselli, G.F.; Yancy, C.W. Referral, enrollment, and delivery of cardiac rehabilitation/secondary prevention programs at clinical centers and beyond: A presidential advisory from the American Heart Association. Circulation 2011, 124, 2951-2960. [CrossRef] [PubMed]

31. Ades, P.A.; Keteyian, S.J.; Wright, J.S.; Hamm, L.F.; Lui, K.; Newlin, K.; Shepard, D.S.; Thomas, R.J. Increasing cardiac rehabilitation participation from 20\% to 70\%: A road map from the Million Hearts Cardiac Rehabilitation Collaborative. Mayo Clin. Proc. 2017, 92, 234-242. [CrossRef] [PubMed]

32. Wang, J.M.; Kim, B.Y.; Bae, J.W.; Oh, D.J. Implementation of national health policy for the prevention and control of cardiovascular disease in South Korea: Regional-local Cardio-Cerebrovascular Center and Nationwide Registry. Korean Circ. J. 2021, 51, $383-398$. [CrossRef]

33. Thomas, R.J.; Beatty, A.L.; Beckie, T.M.; Brewer, L.C.; Brown, T.M.; Forman, D.E.; Franklin, B.A.; Keteyian, S.J.; Kitzman, D.W.; Regensteiner, J.G.; et al. Home-based cardiac rehabilitation: A scientific statement from the American Association of Cardiovascular and Pulmonary Rehabilitation, the American Heart Association, and the American College of Cardiology. J. Cardiopulm. Rehabil. Prev. 2019, 39, 208-225. [CrossRef] [PubMed]

34. Taylor, R.S.; Dalal, H.; Jolly, K.; Zawada, A.; Dean, S.G.; Cowie, A.; Norton, R.J.; Taylor, R.S. Home-based versus centre-based cardiac rehabilitation. Cochrane Database Syst. Rev. 2015, 18, CD007130. 